\title{
miR-125a regulates angiogenesis of gastric cancer by targeting vascular endothelial growth factor $A$
}

\author{
JUN DAI $^{1}$, JINYU WANG $^{2}$, LILI YANG $^{1}$, YING XIAO $^{1}$ and QIURONG RUAN $^{1}$ \\ ${ }^{1}$ Institute of Pathology of Tongji Hospital of Tongji Medical College, Huazhong University of Science \\ and Technology, Wuhan 430030; ${ }^{2}$ Department of Pediatrics, The Second Affiliated Hospital, \\ Zhejiang University College of Medicine, Hangzhou 310009, P.R. China
}

Received June 23, 2015; Accepted August 17, 2015

DOI: 10.3892/ijo.2015.3171

\begin{abstract}
A recent discovery revealed that microRNAs (miRNAs) have an essential effect in the development and progression of gastric cancer (GC). It has already been shown that miR-125a may inhibit tumor development by targeting human epidermal growth factor receptor-2 (Her-2) in GC; however, the other roles of miR-125a in gastric cancer remained to be explored. Our study confirmed that miR-125a was indeed capable of modulating the expression of VEGF-A in gastric cancer cells. In vitro, low expression of miR-125a was able to maintain the secretion of VEGF-A, while the latter increased Akt phosphorylation level in endothelial cells and thereby promoted the proliferation, migration and angiogenesis of human umbilical vein endothelial cells (HUVECs). Our investigation showed that miR-125a expression decreased significantly in gastric cancer comparing with normal gastric tissue and was negatively correlated with the expression of VEGF-A $(\mathrm{P}<0.05)$. In vivo, the expression of miR-125a was inversely proportional to microvessel density (MVD) $(r=-0.5382, \mathrm{P}<0.001)$. The results of this study suggested that low expression of miR-125a predict a worse survival in gastric cancer patients. Collectively, our results indicated that miR-125a regulated the paracrine of VEGF-A in gastric cancer and thereby controlled the angiogenesis of the tumor.
\end{abstract}

\section{Introduction}

GC is a type of malignant tumor with high incidence and mortality (1). Due to the complex pathogenesis of gastric cancer, it lacks more effective treatments. Therefore, clarifying the pathogenesis of gastric cancer is a priority.

Correspondence to: Professor Qiurong Ruan, Institute of Pathology of Tongji Hospital of Tongji Medical College, Huazhong University of Science and Technology, 1095 Jie Fang Avenue, Wuhan 430030, Hubei, P.R. China

E-mail: ruanqiurong@sina.com

Key words: gastric cancer, angiogenesis, miR-125a, VEGF-A
miRNAs are a class of small non-coding single-stranded RNAs. They combine with 3'- or 5'-untranslated region (UTR) of mRNAs in specific target genes to inhibit mRNAs translation or directly degrade their specific mRNAs, involving in regulating a variety of physiological and pathological processes such as cell proliferation, differentiation, apoptosis and metabolism (2-5). miR-125a is located in 19q13, which is frequently downregulated in several human cancers, including breast cancer (6), ovarian cancer (7), lung cancer (8) and medulloblastoma (9). Low expression of miR-125a was associated with the malignant potential indicators of enhanced GC such as tumor size and tumor invasion (10). Survival analysis indicated that low expression of miR-125a was an independent prognostic factor for GC patients, which may be related to the target gene Her-2 (11). However, the mechanism of miR-125a underlying $\mathrm{GC}$ is still unclear.

VEGF-A is considered to be the most potent angiogenic factors, which exerts its effect by activating vascular endothelial growth factor receptor 2 (VEGFR-2). During angiogenesis, VEGF-A binds to VEGFR-2 and activates multiple downstream pathways via signaling intermediates, such as PI3K/Akt (12-16). As a result, VEGF signaling may promote endothelial cell (EC) proliferation, survival, migration, filopodial extension and chemotaxis (17-19). VEGF-A plays an important role in gastric cancer, not only enhancing the malignant potential of tumor cells but also promoting the angiogenesis in the tumors (20-22). Moreover, expression level of VEGF-A in gastric cancer can directly affect the survival of patients (23).

The aim of the present study was to investigate the role of miR-125a in gastric cancer and the mechanism underlying it. We confirmed that miR-125a can function as a crucial tumor suppressor in human gastric cancer. The results showed that miR-125a decreased significantly in gastric cancer and was negatively correlated with the expression of VEGF-A, suggesting that miR-125a may inhibit angiogenesis of gastric cancer by downregulating VEGF-A.

\section{Materials and methods}

Patients. Consecutive series of 73 cases of gastric cancer and 20 cases of normal gastric tissues specimens were collected from Institute of Pathology of Tongji Hospital, Huazhong 
University of Science and Technology in 2009. Patients with a previous history of another primary tumor, or those who had previously received chemotherapy and/or radiotherapy were excluded from the study. As to the diagnosis of gastric cancer, tumors were analyzed and discussed by two pathologists, and then a definite diagnosis was made. The age, sex, tumor size, lymph node (LN) metastasis and clinical stage of the patients were recorded.

Cell culture. Cell lines GES-1, AGS, SGC7901, BCG-823 and HUVEC were purchased from China Center for type culture collection in Wuhan. All cells were cultured with RPMI-1640 medium (Gibco, USA) containing $10 \%$ fetal bovine serum (FBS) (Gibco), while instructions were followed additionally under special circumstances.

Cell transfection. AGS cells were grown in 6-well plates to reach a cell density of $60 \%$ the next day. Lipofectamine 2000 (Invitrogen, USA) was mixed with miR-125a mimics and miR-125a inhibitors (GenePharma, China) respectively for $10 \mathrm{~min}$. After washing cells twice with serum-free opti-MEM medium, appropriate amount of opti-MEM was added to each well, then the above mixture was added to the medium dropwise to reach a concentration of $40 \mathrm{nM}$. The cells were incubated at $37^{\circ} \mathrm{C}$ for $6 \mathrm{~h}$, then the mixture was replaced with medium containing $10 \%$ FBS.

QRT-PCR. For miRNAs, in vivo, formalin-fixed paraffinembedded tissue (FFPET) samples were cut into $10-\mu \mathrm{m}$ thin slices, with a total thickness of $40 \mu \mathrm{m}$. miRNAs from FFPET was extracted in accordance with the manufacturer's protocol (Aidlab, RN3101, China). In vitro, total RNA was extracted from AGS cells using TRIzol (Invitrogen). U6 snRNA was used as internal reference for qRT-PCR. Hsa-miR-125a-5p and U6 specific primers as well as the miRNA qRT-PCR Detection kit were purchased from GeneCopoeia. For mRNAs, total cellular RNA was extracted with TRIzol (Invitrogen). With random primer dNTP (Fermentas, R0191) and revertAid reverse transcriptase (Fermentas, EP0442), mRNA was reversely transcribed to cDNA. The PCR primers were as follows: VEGF-A forward, 5'-CGAAGTGGTGGTCATGGATG-3'; reverse, 5'-TTCTGTATCAGTCTTTCCTGGTGA-G-3'; GAPDH forward, 5'-AGGTCGGAGTCAACGGATTTG-3'; reverse, 5'-GTGATGGCA-TGGACTGTGGT-3'. Real-time PCR was performed using the ABI7500 real-time by Applied Biosystems.

Western blotting. Total cellular protein was collected using RIPA lysis buffer (Beyotime, China). Bicinchoninic acid (BCA) Protein Assay kit (Pierce, USA) was used to quantify the protein concentration. Equal amounts of protein were loaded into SDS-polyacrylamide gels and transferred onto polyvinylidene difluoride filter (PVDF) membrane. After blocked with $5 \%$ skim milk for $1 \mathrm{~h}$, the membranes were incubated with VEGF-A antibody (Santa Cruz, USA), Akt antibody and p-Akt antibody (Cell Signaling Technology, USA) respectively, at $4^{\circ} \mathrm{C}$ overnight. The next day, the membranes were incubated with HRP-conjugated secondary antibody (Santa Cruz) for $1 \mathrm{~h}$ at $37^{\circ} \mathrm{C}$ and then detected by chemiluminescence system (Beyotime).
ELISA. Forty-eight hours after transfection, the old medium was replaced with serum-free medium and cells were incubated for another $24 \mathrm{~h}$. Then the medium was collected to detect the VEGF-A concentration by ELISA assay (Abcam, USA) according to the manufacturer's protocol. Thereafter, the serum-free medium containing different concentrations of VEGF-A (AGS-VEGF-A-medium) was placed on standby at $-80^{\circ} \mathrm{C}$ for $\mathrm{EC}$ proliferation and tube formation assays (24).

Luciferase reporter assay. First, we predicted that human miR-125a was able to regulate the expression of VEGF-A, and we aimed to identify the possible site (http://www.microrna. org). The 3'-UTR of human VEGF-A was amplified by PCR (25). The primers for PCR amplification are: forward, 5'-ATCTCAGCATGCCTGGTCAGTTACCTACTAATAGC GGGCCTG-3' and reverse: 5'-GCCCTGAGTGCTGAGC GATCAAGTGTCATTTGACGTATCGCT-3'. Then the amplified sequence was cloned into the $X b a I$ site of the pGL3 control vector (Promega, USA). The mutated putative miR-125a-5p binding site in the 3'-UTR of VEGF-A was generated using the QuickChange Site-directed Mutagenesis kit (Stratagene, Cedar Creek) according to the manufacturer's protocol. The day before transfection, AGS cells were seeded in 24-well plates $\left(5 \times 10^{4}\right.$ cells/well), then we transfected $500 \mathrm{ng}$ VEGF-A3'-UTR-pGL3 and $500 \mathrm{ng}$ miR-125a mimics or control miRNA mimics (GenePharma, China) into cells using Lipofectamine 2000. Forty-eight hours after transfection, luciferase activity was determined using dual-luciferase reporter assay system (Promega) according to the manufacturer's protocol.

Cell proliferation assay. Cell proliferation assays were performed using HUVEC essentially as described (26-28). HUVECs were resuspended to a density of $2 \times 10^{4} / \mathrm{ml}$. For CCK-8 proliferation assay, $100 \mu \mathrm{l}$ cell suspension was planted into each well of 96-well plates. The medium was replaced after cell adherence. The AGS-VEGF-A-medium or VEGF-Amedium (VEGF-A recombinant protein, $3 \mathrm{ng} / \mathrm{ml}$, Sino Biological Inc., China) was formulated as conditioned medium containing $2 \% \mathrm{FBS}$, and Akt activity inhibitor (MK-22062HCI, $10 \mu \mathrm{M}$, Selleck, USA) and used to conduct control experiment. The cell proliferation activity was detected using cell counting kit-8 (CCK8) (Beyotime). After cultured for a few days, $10 \mu \mathrm{l}$ CCK8 was added to each well and incubated for $1 \mathrm{~h}$ in the cell incubator. The optical density (OD) value was read at $450 \mathrm{~nm}$ wavelength. For plate colony formation assay, $1 \times 10^{3}$ cells were seeded into 6-well plates and cultured for 2 weeks. The colonies were fixed with $4 \%$ paraformaldehyde for $10 \mathrm{~min}$ and stained with $0.1 \%$ crystal violet (Beyotime) for $5 \mathrm{~min}$. Clones with $>50$ cells were counted $(29,30)$. Experiments were repeated at least 3 times.

Cell migration assay. HUVEC migration was evaluated with a transwell system (Corning Costar, USA) that comprised $8-\mu \mathrm{m}$ inserts in 24-well plates. Cells were co-cultured as described $(31,32)$. Firstly, genetically modified AGS cells or VEGF-A-medium $(3 \mathrm{ng} / \mathrm{ml})$ were added into the lower chamber. When AGS cell fusion reached to $100 \%$, the growth medium was replaced with serum-free medium. HUVECs were resuspended in serum-free medium with $0.1 \%$ bovine 
A

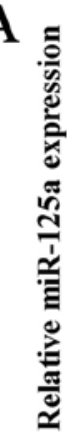

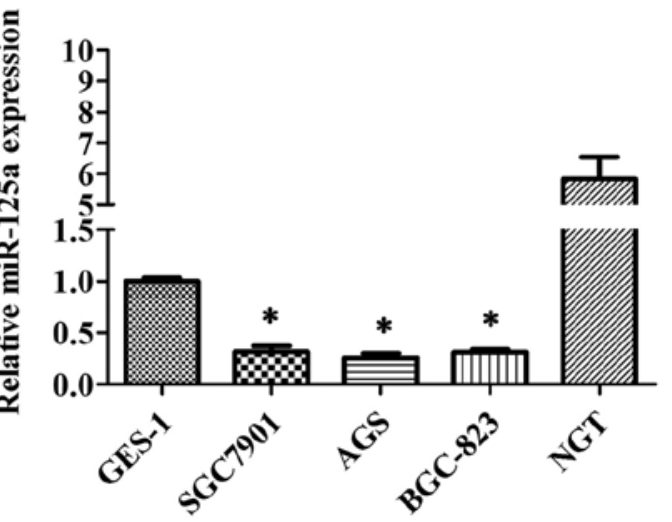

B

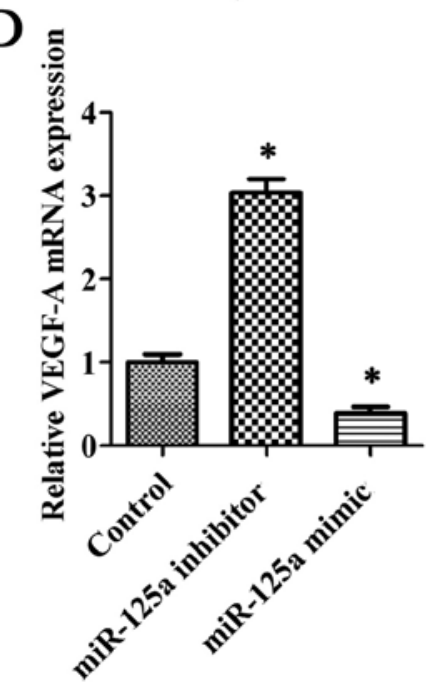

B

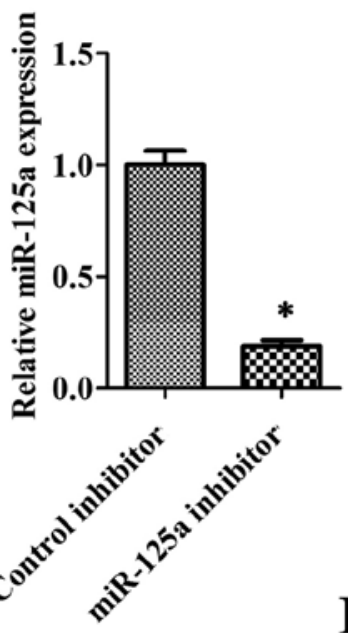

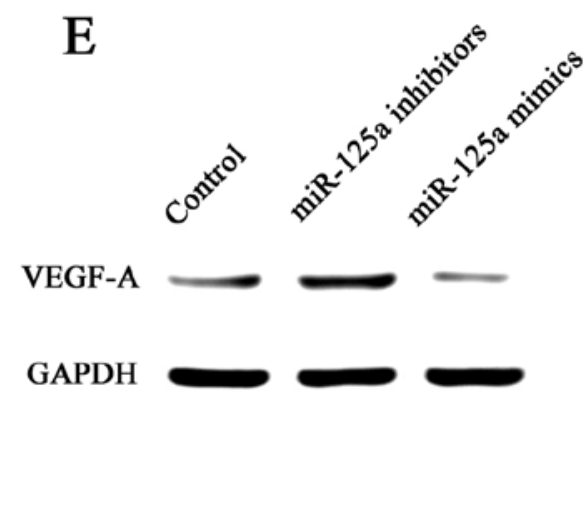

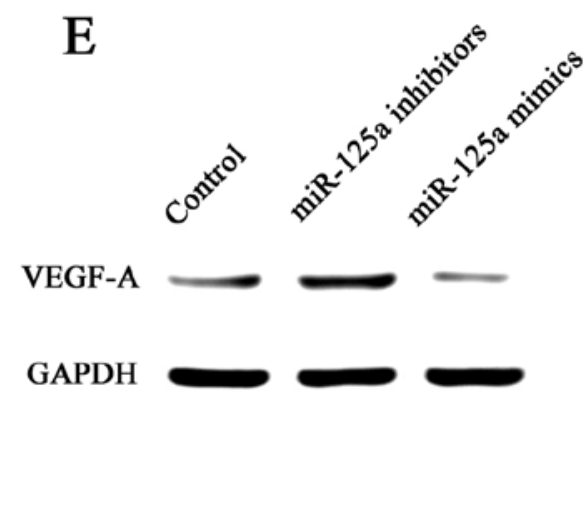

C

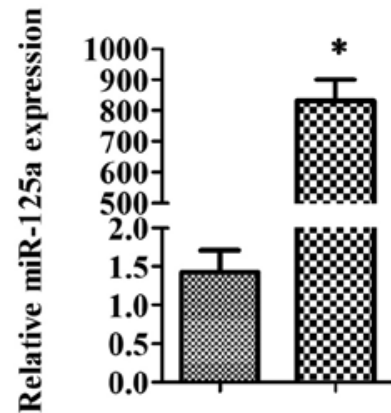

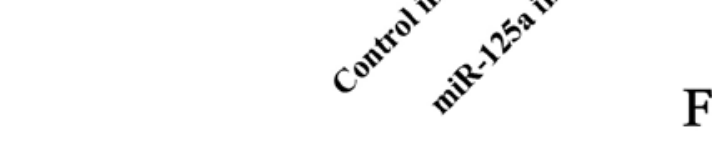

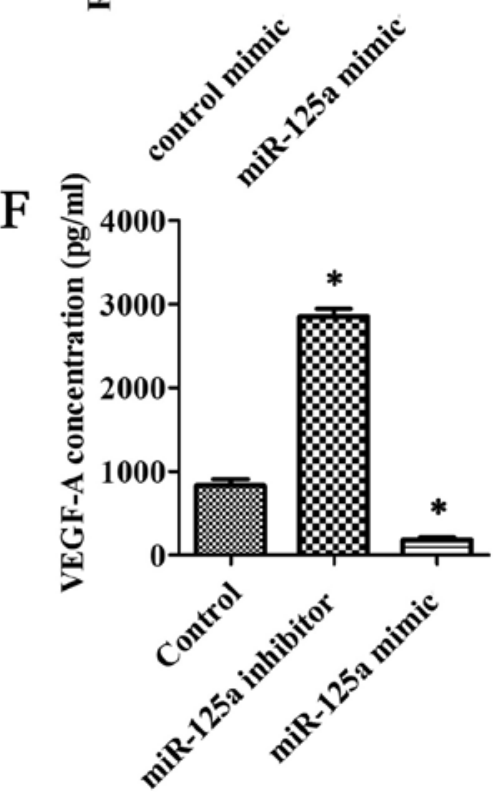

Figure 1. Regulation of the expression of VEGF-A by miR-125a. (A) The relative expression of miR-125a in different cell lines and normal gastric tissue (NGT). (B and C) After transfected with miR-125a inhibitors or miR-125a mimics, the expression of miR-125a was analyzed by qRT-PCR in AGS. (D-F) The changes of VEGF-A mRNA and protein expression after transfecting miR-125a inhibitors or mimics into AGS cells. "P<0.05 vs. control group.

serum albumin (BSA) to a density of $5 \times 10^{5} / \mathrm{ml}$. Then $100 \mu \mathrm{l}$ medium containing HUVECs were added in each upper chamber (presence or absence of Akt inhibitor). After incubation for $24 \mathrm{~h}$, cells that did not migrate in the upper chamber were removed with a cotton swab. Then the migrated cells were fixed with $4 \%$ paraformaldehyde for $20 \mathrm{~min}$. After that, the cells were stained with $0.1 \%$ crystal violet (Beyotime) for $5 \mathrm{~min}$ and counted and photographed with a fluorescence microscope on x100 magnification. Experiments were repeated at least 3 times.

In vitro tube formation assay. In vitro tube formation assays were performed using HUVEC essentially as described $(33,34)$. Briefly, $50 \mu 1$ growth factor-reduced Matrigel (BD, USA), which was thawed on ice in advance, were plated in 96-well plates and incubated at $37^{\circ} \mathrm{C}$ for $45 \mathrm{~min}$. In order to investigate the effect of AGS-VEGF-A-medium or VEGF-A-medium (absence of FBS, presence or absence of Akt inhibitor) on angiogenesis in HUVECs, the cells $\left(100 \mu 1,2 \times 10^{5}\right.$ cells $\left./ \mathrm{ml}\right)$ were seeded on the matrigel and incubated at $37^{\circ} \mathrm{C}$ with $5 \% \mathrm{CO}_{2}$ for $4 \mathrm{~h}$. After the culture media were removed, the cells were washed twice with PBS. Then stained with $0.1 \%$ crystal violet for $5 \mathrm{~min}$ and washed with PBS twice again. Images were captured using a digital camera (x100 magnification). Tubes and nodal structures were counted by two independent researchers. Experiments were repeated at least 3 times.

Immunohistochemistry (IHC). The tumor specimens were fixed, dehydrated and embedded in paraffin, then cut into $3-\mu \mathrm{m}$ thin slices. After dewaxed and rehydrated, they were autoclaved for 2 min and then incubated with 3\% hydrogen peroxide for $10 \mathrm{~min}$ at room temperature to remove endogenous peroxidase activity. The slices were treated with 5\% BSA for $30 \mathrm{~min}$, followed by incubating with Her-2 antibody (ZSGB-BIO, China) and VEGF-A antibody (Santa Cruz) at $4^{\circ} \mathrm{C}$ overnight, respectively, then washed in phosphatebuffered saline (PBS) for 2 min and incubate with secondary antibodies (Dako, USA) at $37^{\circ} \mathrm{C}$ for $1 \mathrm{~h}$. Then staining was with diaminobenzidine (DAB) substrate chromogen solution for $5 \mathrm{~min}$ at room temperature. Her- 2 was evaluated by two pathologists independently. Her-2(0), Her-2(1+) and Her-2(2+) were defined as Her-2(-), while Her-2(3+) was defined as Her-2(+). The evaluation of VEGF-A was analyzed using ImageJ software (National Institutes of Health, USA). The raw data was adjusted appropriately to gain normalized score, and the normalized score greater than 2 was designated as VEGF-A positive, while the other negative. 
A

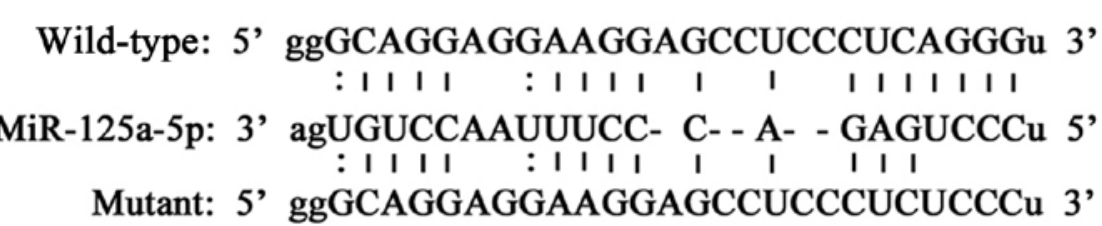

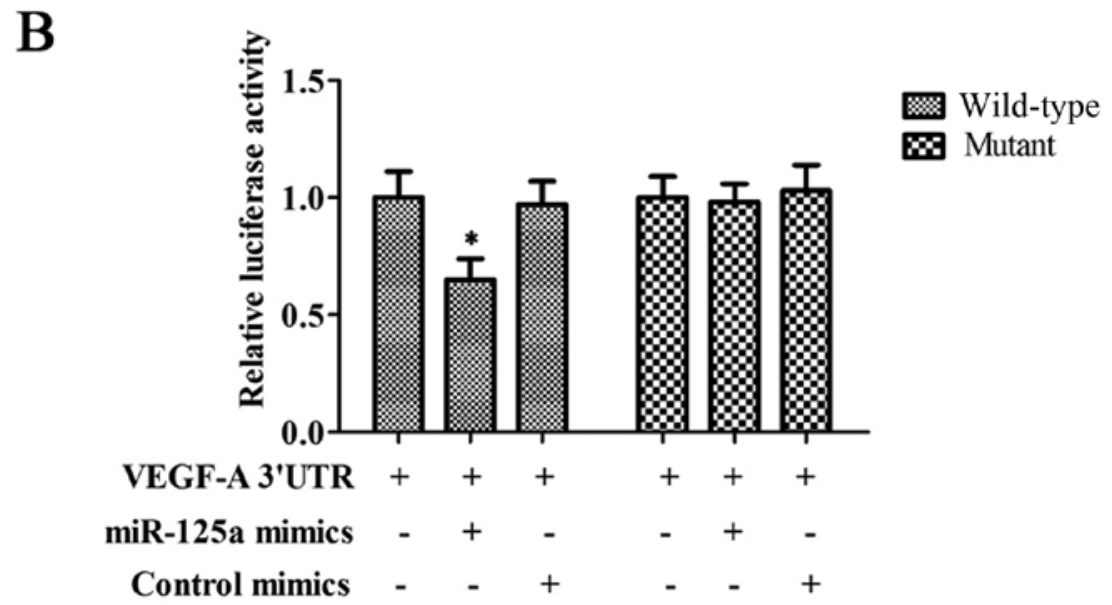

Figure 2. VEGF-A is a target gene of miR-125a. (A) Binding sites of miR-125a in VEGF-A was predicted. (B) The luciferase reporter assay revealed that miR-125a can result in a significant reduction of the relative luciferase activity while mutating the binding sites abolished this effect. ${ }^{*}<0.05$ vs. control group.

MVD analysis. CD31 (Santa Cruz) was detected using IHC staining methods in 73 cases of FFPET to observe MVD in gastric cancer. Evaluation of MVD was performed by two authors independently. MVD in the tumor was determined as described $(35,36)$. In brief, areas rich in tumor tissue and blood vessels were selected at low magnification (x40). Then the blood vessels were counted in the region at high magnification (x100), or even a higher magnification (x200) for some individual vessels. A single independent CD31 staining cell, or microvessel that had formed tubular structure was considered as a countable microvessel. In order to reflect the MVD more accurately, we kept count of five horizons (x100) accumulatively.

Statistical analysis. The experimental data were presented as mean \pm SD. The differences among variables were assessed by $\chi^{2}$ analysis or 2-tailed Student's t-test. Survival curves were plotted by the Kaplan-Meier method, and differences were assessed by the log-rank test. Correlation parameters were submitted to Pearson's correlation. Statistical analyses were conducted using GraphPad Prism 5.0 (GraphPad Software Inc., San Diego, CA, USA). $\mathrm{P}<0.05$ was considered to have a significant statistical difference.

\section{Results}

miR-125a regulates the expression of VEGF-A in GC cells. QRT-PCR analysis showed that miR-125a expression in gastric cancer cells (SGC7901, AGS and BGC-823) were lower than that in GES-1 cells or normal gastric tissue (NGT) (Fig. 1A). We transfected miR-125a inhibitors or miR-125a mimics into AGS cells (Fig. 1B and C). Our investigations revealed that the
VEGF-A mRNA levels in groups transfected with miR-125a mimics were significantly lower than the control group; in contrast, the VEGF-A mRNA levels were significantly higher in groups transfected with miR-125a inhibitors (Fig. 1D). On the other hand, VEGF-A protein levels detected by western blotting were also reduced in groups that transfected with miR-125a mimics and increased in groups transfected with miR-125a inhibitors (Fig. 1E). Moreover, we obtained a similar result when compared VEGF-A protein levels in the medium of the transfected groups with that in control groups using ELISA technique (Fig. 1F).

$V E G F-A$ is a target gene of miR-125a. Via predictive analysis, we found that miR-125a had binding sites in the 3'-UTR region of VEGF-A mRNA (Fig. 2A). To verify whether miR-125a was capable of regulating the expression of VEGF-A, we performed a luciferase reporter assay. The luciferase reporter assay revealed that miR-125a can result in a significant reduction of the relative luciferase activity while mutating the binding sites abolished this effect (Fig. 2B).

Enhanced migration potential of HUVECs due to increased secretion of VEGF-A in gastric cancer cells. Co-culture transwell assay was used to detect the migration ability of HUVECs. The migration ability of HUVECs declined when HUVECs were co-cultured with miR-125a mimic transfected AGS cells, while the capacity was enhanced in miR-125a inhibitor transfection system. However, this effect can be repealed by Akt inhibitor MK-2206. Moreover, VEGF-A recombinant protein may contribute to the migration of HUVECs. Similarly, this effect can be abolished by MK-2206 (Fig. 3). 
A

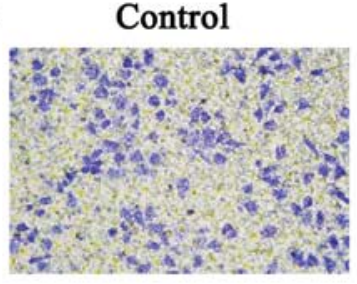

miR-125a inhibitor

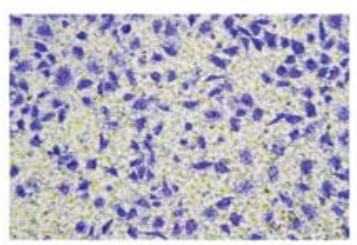

VEGF-A

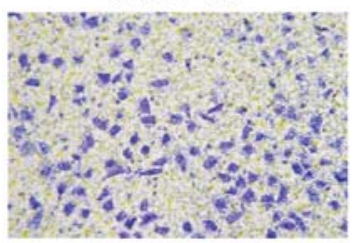

miR-125a mimic

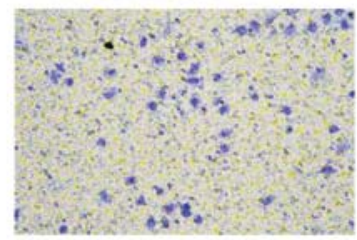

inhibitor + MK-2206

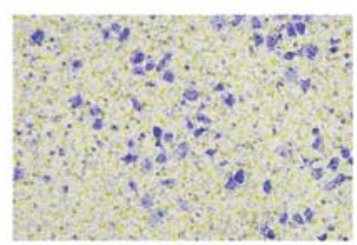

VEGF-A + MK-2206

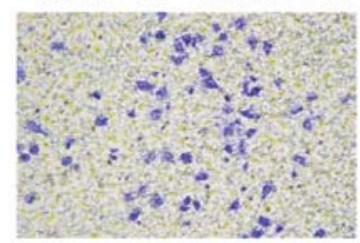

B

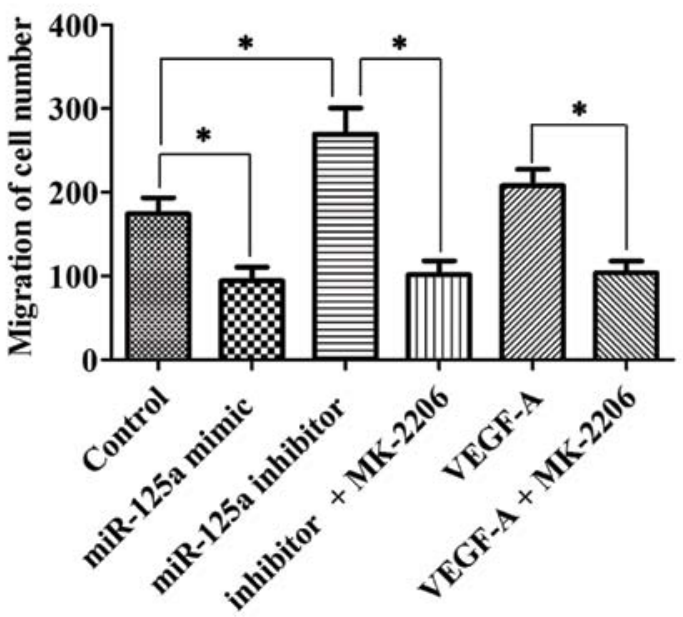

Figure 3. VEGF-A promotes the migration of HUVECs. (A) Transwell assay was used to detected the migration ability of HUVECs in different groups. (B) Compare of the migration abiliy of HUVECs in different groups. " $\mathrm{P}<0.05$ vs. control group.

Increased secretion of VEGF-A in gastric cancer cells may enhance the angiogenesis potential of HUVECs. In vitro tube formation assay was peformed to detect the angiogenesis potential of HUVECs in different conditioned media. Comparing conditioned medium of control AGS cells with that of miR-125a mimic-transfected AGS cells, the latter contained lower VEGF-A and decreased tube formation (Fig. 4A and B) and nodal structure (Fig. 4A and C); however, conditioned medium of miR-125a inhibitor transfected AGS cells improved the ability of HUVECs to form tube and nodal structures and this effect can be inhibited by Akt inhibitor MK-2206 (Fig. 4A and B). Moreover, exogenous recombinant VEGF-A protein enhanced the tube and nodal structure forming ability of HUVECs, similarly to that shown by MK-2206 (Fig. 4A and C).
Increased secretion of VEGF-A in gastric cancer cells may enhance the proliferation potential of HUVECs. The proliferation potential of endothelial cells directly affected angiogenesis; therefore, it was also necessary to detect changes in the proliferation ability of endothelial cells in conditioned medium. We cultured HUVECs with AGS-VEGF-A-medium or VEGF-A-medium containing different concentrations of VEGF-A. The colony formation assay and CCK-8 proliferation assay revealed significant changes in the proliferation capacity of HUVECs. Increased VEGF-A in media can promote the proliferation of HUVECs, similarly, this effect can be abolished by the Akt inhibitor (Fig. 5A and B). The change in biological function of HUVECs may be associated with the VEGF-A concentration in ambient environment. Increased VEGF-A may contribute to the migration, proliferation and angiogenesis through activation of Akt signaling pathway in endothelial cells, while reduced VEGF-A or inhibition of Akt activity decreased the ability (Fig. 5C).

Relationship between the expression of miR-125a, VEGF-A and MVD in gastric cancer tissues. QRT-PCR was performed to detect the relative expression of miR-125a in GCT and NGT. We found that the expression levels of miR-125a in GCT were lower than that in NGT, which showed a significant statistical difference $(\mathrm{p}<0.05)$ (Fig. 6A). The relative expression of miR-125a in tumors scored $<1.00$ were classified as low expression, while the other cases as high expression. According to this artificial demarcation, the low expression ratio of miR-125a in these 73 cases of gastric cancer tissues was $50.7 \%$. Furthermore, we analyzed the expression of Her-2 and VEGF-A in 73 cases of gastric cancer tissues by IHC. Her-2 was expressed on the membrane of the tumor cells, with a positive rate of $17.8 \%$ (Fig. 6B and C). VEGF-A cytoplasmic expression showed positive rate of $31.5 \%$ (Fig. 6D and E). In order to reveal the relationship between the expression of miR-125a, VEGF-A and the numbers of tumor microvessels in gastric cancer, we performed IHC staining of vascular marker CD31 in 73 cases of gastric cancer tissues. Single independent endothelial cells (red arrow) or microvessels that had formed a complete tubular structure (black arrow) was considered as a countable microvessel (Fig. 6F and G). Correlation analysis suggested that the expression of miR-125a was inversely proportional to MVD ( $\mathrm{r}=-0.5382$ ) (Fig. 6H), while VEGF-A expression was positively related to MVD (r=0.7226) (Fig. 6I). Moreover, the expression of miR-125a and VEGF-A also showed an inverse correlation $(r=-0.4554)$ (Fig. 6J).

Correlation analysis between miR-125a and clinicopathologic parameters in gastric cancer. We analyzed the correlation between miR-125a and clinicopathological parameters in gastric cancer (Table I). The expression of miR-125a was correlated with lymph node status, the MVD of GC tissues, the clinical stage of GC and the expression of VEGF-A and Her-2 $(\mathrm{p}<0.05)$. The clinical significance of miR-125a and VEGF-A in GC was further analyzed based on follow-up of the patients. The low expression of miR-125a indicated a worse prognosis in patients compared to the high expression of miR-125a (Fig. 7A), while low expression of VEGF-A indicated a better prognosis compared to the high expression of VEGF-A (Fig. 7B). MVD was divided into low-density group 
A

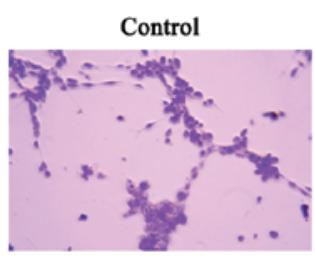

miR-125a inhibitors

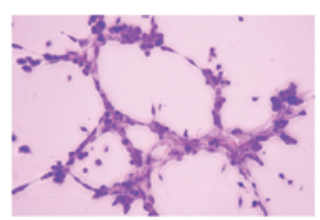

VEGF-A

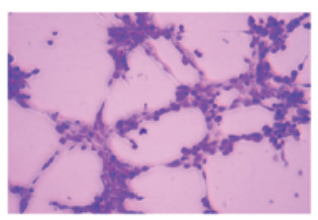

miR-125a mimics

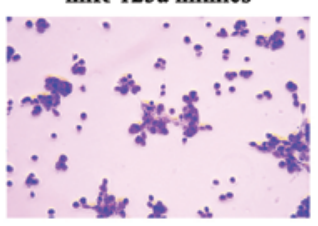

inhibitors + MK-2206

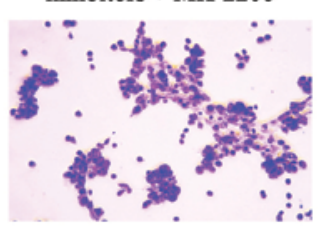

VEGF-A + MK-2206

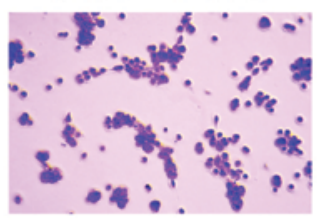

B
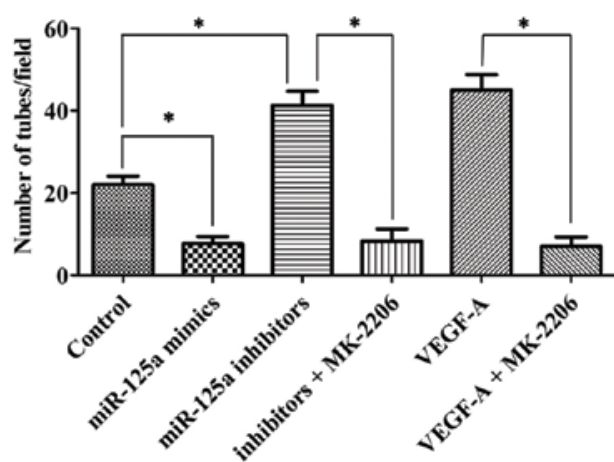

C

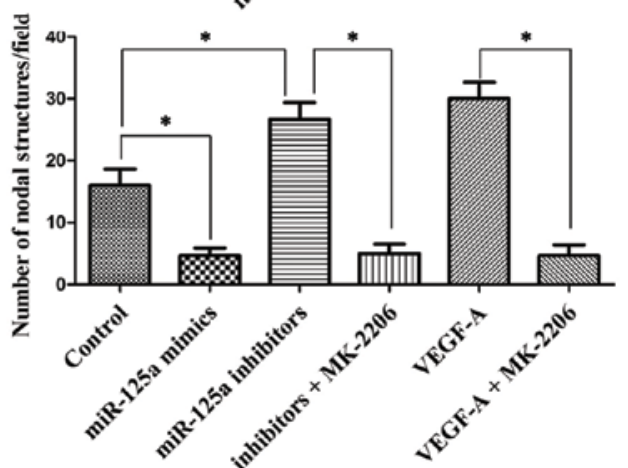

Figure 4. VEGF-A promotes the angiogenesis potential of HUVECs. (A) In vitro tube formation assay of the angiogenesis ability of HUVECs in the different groups. (B) Comparison of the ability of HUVECs tube formation in different groups. (C) Comparison of the ability of HUVECs nodal structure formation in different groups. ${ }^{*} \mathrm{P}<0.05$ vs. control group.

A

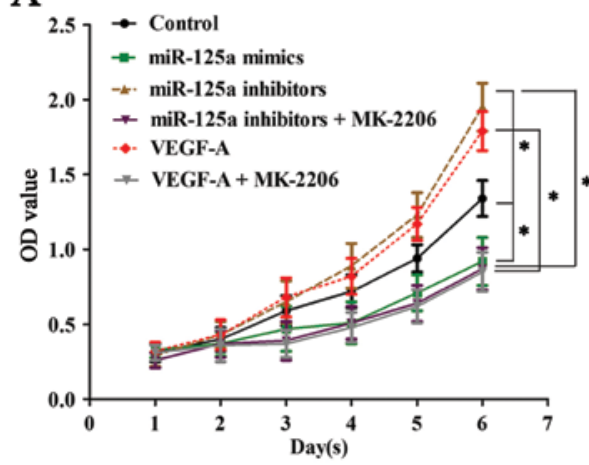

C

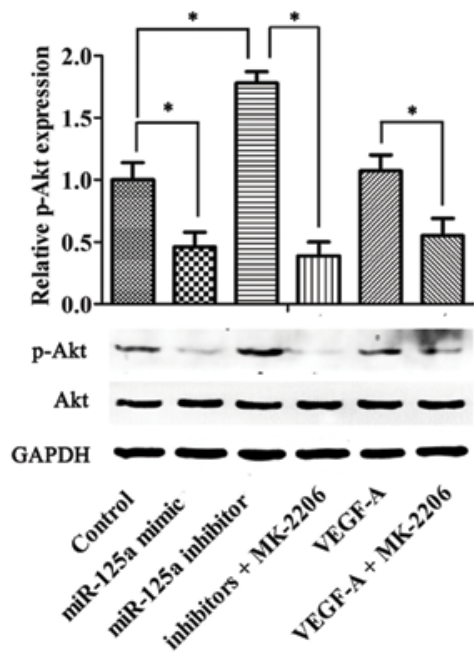

B
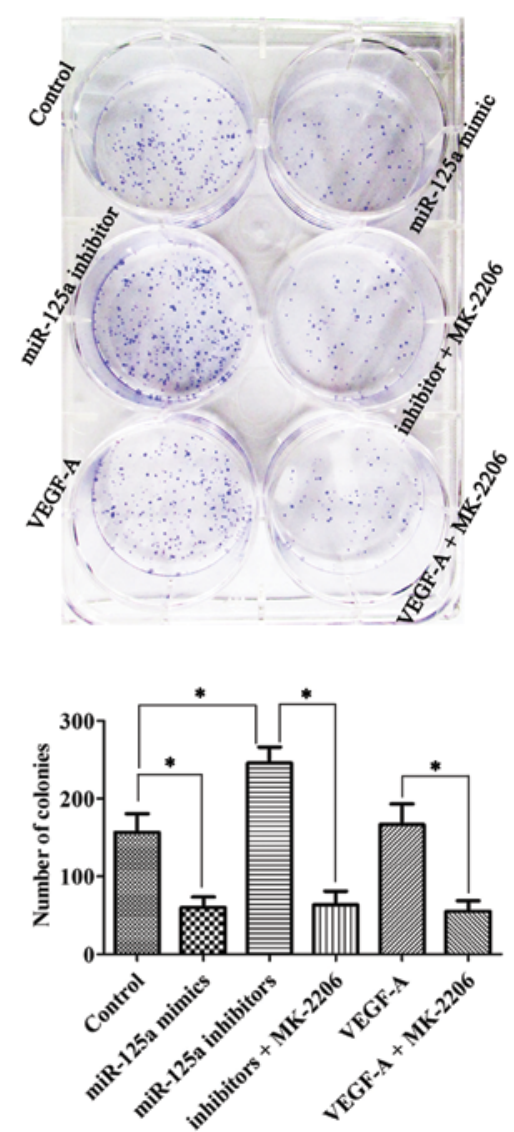

Figure 5. VEGF-A promotes the proliferation of HUVECs. (A) CCK-8 proliferation assay of the changes in proliferation capacity of HUVECs after cultured with VEGF-A-medium containing different concentrations of VEGF-A. (B) Colony formation assay of the changes in proliferation capacity of HUVECs after cultured with VEGF-A-medium containing different concentrations of VEGF-A. (C) Effect of medium containing different concentrations of VEGF-A on Akt phosphorylation of HUVECs. ${ }^{*} \mathrm{P}<0.05$ vs. control group. 

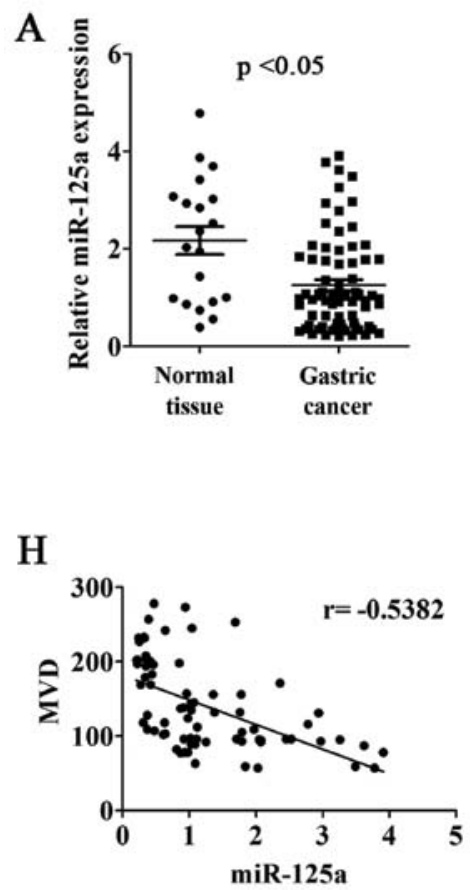

I

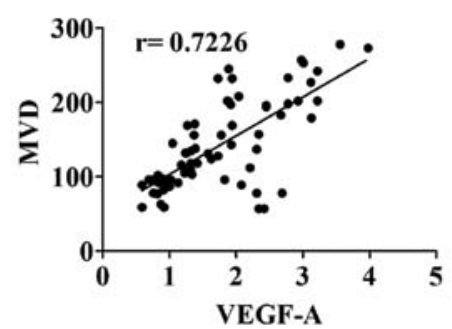

B

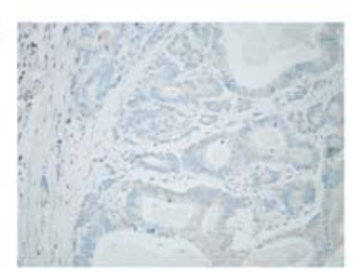

D
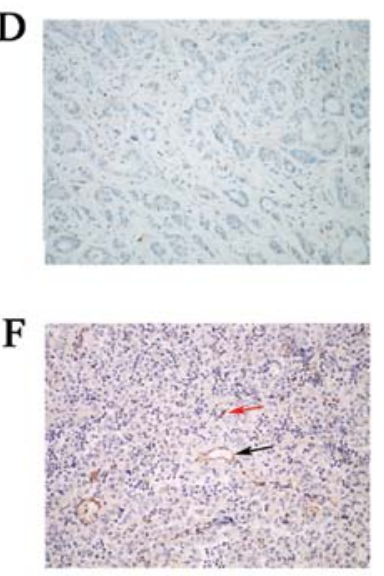

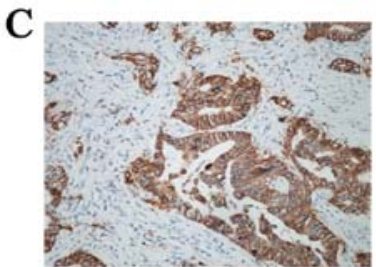

$\mathrm{E}$
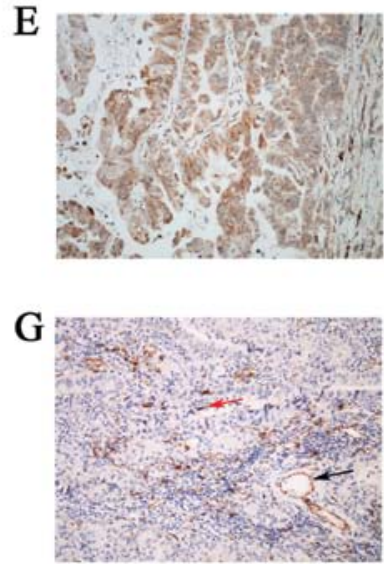

$\mathbf{J}$

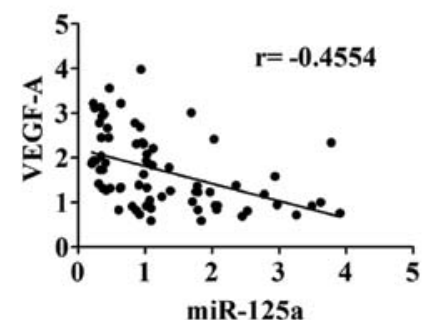

Figure 6. Relationship between the expression of miR-125a, VEGF-A and MVD in GC tissues. (A) miR-125a expression in GCT and NGT. (B) Her-2 negative expression in GCT. (C) Her-2 positive expression in GCT. (D) VEGF-A negative expression in GCT. (C) VEGF-A positive expression in GCT. (F) GCT with low MVD. (G) GCT with high MVD. (H-J) Correlation analysis between miR-125a, VEGF-A and MVD.

A

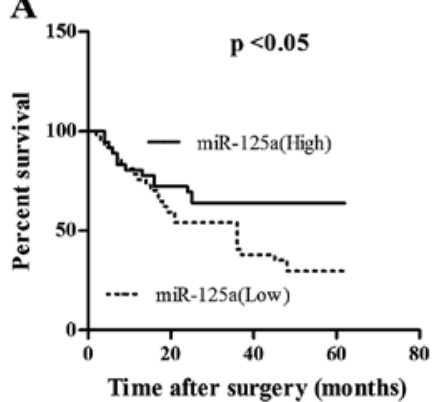

B

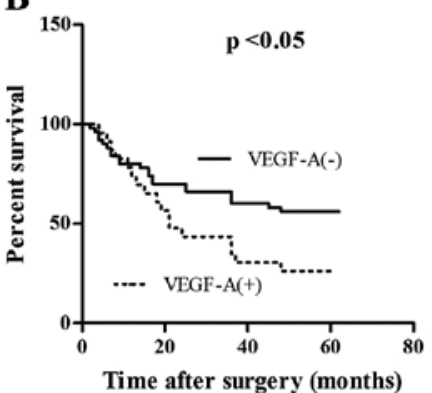

C

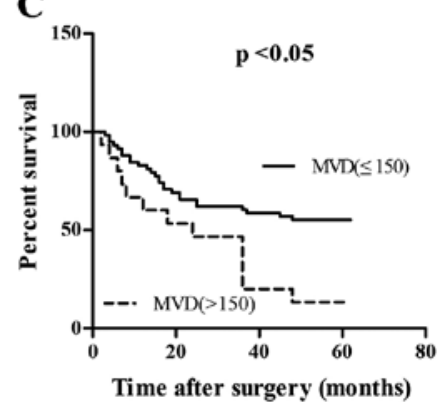

Figure 7. (A) Significant difference in overall survival between low expression and high expression of miR-125a was found. (B) Overall survival time of patients with high expression of VEGF-A was significantly shorter than that of patients with low expression. (C) Significant difference in overall survival between low MVD and high MVD in GC patients.

and high-density group when 150 was set as the critical value. The results indicate that high-density group had a poorer prognosis (Fig. 7C).

\section{Discussion}

miRNAs have been shown to play important roles in the development of tumors by participating in regulation of gene expression
(37-40). miRNAs have been reported as potential new tumor markers in recent years. Therefore, exploring the clinical roles and molecular mechanisms of miRNAs in the malignant development is an effective way to delay the development of GC and improve the prognosis of GC patients. In this study, we mainly investigated the role of miR-125a in angiogenesis in GC.

Substantial evidence has suggested that higher density of tumor blood vessels predict worse prognosis of cancer 
Table I. Correlation of miR-125a and the clinicopathological parameters.

\begin{tabular}{|c|c|c|c|}
\hline \multirow[b]{2}{*}{$\begin{array}{l}\text { Clinicopathological } \\
\text { parameters }\end{array}$} & \multicolumn{3}{|c|}{ miR-125a } \\
\hline & Low & High & P-value \\
\hline \multicolumn{4}{|l|}{ Age (years) } \\
\hline$\leq 50$ & 16 & 18 & 0.563 \\
\hline$>50$ & 21 & 18 & \\
\hline \multicolumn{4}{|l|}{ Gender } \\
\hline Male & 18 & 16 & 0.719 \\
\hline Female & 19 & 20 & \\
\hline \multicolumn{4}{|l|}{ Size $(\mathrm{cm})$} \\
\hline$\leq 5$ & 20 & 23 & 0.393 \\
\hline$>5$ & 17 & 13 & \\
\hline \multicolumn{4}{|l|}{ LN status } \\
\hline Negative & 7 & 16 & $0.019^{\mathrm{a}}$ \\
\hline Positive & 30 & 20 & \\
\hline \multicolumn{4}{|l|}{ MVD } \\
\hline$\leq 150$ & 24 & 34 & $0.002^{\mathrm{a}}$ \\
\hline$>150$ & 13 & 2 & \\
\hline \multicolumn{4}{|l|}{ TNM } \\
\hline I, II & 9 & 22 & $0.001^{\mathrm{a}}$ \\
\hline III, IV & 28 & 14 & \\
\hline \multicolumn{4}{|l|}{ Her-2 } \\
\hline $0-2+$ & 26 & 34 & $0.007^{\mathrm{a}}$ \\
\hline $3+$ & 11 & 2 & \\
\hline \multicolumn{4}{|l|}{ VEGF-A } \\
\hline Negative & 19 & 31 & $0.001^{\mathrm{a}}$ \\
\hline Positive & 18 & 5 & \\
\hline
\end{tabular}

LN, lymph node; TNM, tumor node metastasis.

patients $(36,41,42)$. Rich blood vessels are the basis for abnormal growth of the tumor, while the paracrine amount of pro-angiogenic factors by tumor cells is an important link to decide the angiogenesis potential of tumor (43-46). VEGF is a class of endothelium-specific growth factor, which plays an important role in the development of tumor angiogenesis. There are seven members in VEGF families, including VEGF-A, -B, -C, -D, -E, -F and placental growth factor (47). The activation of VEGF-A/VEGFR-2 pathway is sufficient to promote the EC proliferation, migration and tube formation. VEGF-A/VEGFR-2 can promote the tumor growth of both primary and metastatic cancer, indicating that VEGF-A plays a key role in tumor angiogenesis (48). This study demonstrated that the growth of GC was attributable to the overexpression of VEGF-A by facilitating angiogenesis, as the expression of VEGF-A was positively related to microvessel quantity in tumor tissues. In vivo, MVD analysis showed that the number of microvessels in VEGF-A-positive GC had a statistically significant difference compared to VEGF-A-negative GC. In vitro, gastric cancer cells were transfected with miR-125a mimics or miR-125a inhibitors, resulting in the reduction or increase of VEGF-A secretion. Then the medium containing different concentrations of VEGF-A were co-cultured with HUVECs, which may change the proliferation, migration and angiogenesis ability of HUVECs at least partly by VEGF-A/ VEGFR2/PI3K/Akt signaling pathway $(12,16,49,50)$.

miR-125a was an independent prognostic factor in gastric cancer, which may be related to the target gene Her-2 $(10,11)$. Ectopic expression of miR-125a-5p substantially inhibited the proliferation, migration and invasion by targeting E3F3 in gastric cancer cells (51). It has also been reported that miR-125a may inhibit the proliferation and metastasis of hepatocellular carcinoma partly by downregulating MMP11 and VEGF-A (52). However, the relative contribution of miR-125a in regulating VEGF-A in gastric cancer remained unknown. This study demonstrated that miR-125a decreased significantly in gastric cancer. Moreover, the expression of miR-125a was associated with the clinical stage of gastric cancer and the expression of VEGF-A, suggesting that it may become a new biological marker. Further study showed that the expression levels of VEGF-A in the groups transfected with miR-125a mimics were significantly lower than the control groups, which indicated that miR-125a may downregulate the expression of VEGF-A in gastric cancer. This conclusion was corroborated by our luciferase reporter assay, which showed that VEGF-A was a direct target gene of miR-125a in gastric cancer. VEGF-A secreted by gastric cancer cells acted on vascular endothelial cells around the tumor, promoting the proliferation, and migration of the endothelial cells, and thereby enhancing angiogenesis. Combining correlation analysis between miR-125a, VEGF-A and MVD with in vitro results, we hypothesized that miR-125a/ VEGF-A signaling pathway may be a new approach to regulate angiogenesis in GC. Gastric cancer metabolize vigorously. High-density microvascularity can provide a wealth of nutrients for tumor cells, transport the metabolites and thereby promote the development of the tumor. The follow-up study showed that miR-125a, VEGF-A, and MVD in tumor tissues can all predict the prognosis in patients. In fact, anti-angiogenesis approach has already been used to treat cancer in recent years. Although it has certain effects, many deficiencies exist $(43,53,54)$. Theoretically, any molecule in miR-125a/VEGF-A/VEGFR2/Akt signaling pathway may become a therapeutic target. However, antiangiogenic drugs targeting VEGF or its receptors, which aim at blocking the blood supply to tumors, may also cause hypoxia of normal body and thereby fuel tumor progression and treatment resistance. Perhaps targeting the upstream molecules of VEGF-A signaling, such as miR-125a, can achieve a therapeutic effect; however, there is a long way to go yet.

In conclusion, this study provided evidence that miR-125a inhibited the growth of gastric cancer by targeting VEGF-A. Furthermore, increased levels of miR-125a resulted in inhibition of angiogenesis in gastric cancer. Taken together, these findings may help to understand the pathogenesis of gastric cancer in depth and bring new ideas to the treatment of gastric cancer in the future.

\section{Acknowledgements}

This study was supported by grants from Natural Science Foundation of China (no. 30570725). 


\section{References}

1. Jemal A, Bray F, Center MM, Ferlay J, Ward E and Forman D: Global cancer statistics. CA Cancer J Clin 61: 69-90, 2011.

2. Bartel DP: MicroRNAs: Genomics, biogenesis, mechanism, and function. Cell 116: 281-297, 2004.

3. Ambros V: The functions of animal microRNAs. Nature 431: 350-355, 2004.

4. Lujambio A and Lowe SW: The microcosmos of cancer. Nature 482: 347-355, 2012.

5. Bartel DP: MicroRNAs: Target recognition and regulatory functions. Cell 136: 215-233, 2009.

6. O'Day E and Lal A: MicroRNAs and their target gene networks in breast cancer. Breast Cancer Res 12: 201, 2010.

7. Nam EJ, Yoon H, Kim SW, Kim H, Kim YT, Kim JH, Kim JW and Kim S: MicroRNA expression profiles in serous ovarian carcinoma. Clin Cancer Res 14: 2690-2695, 2008.

8. Jiang L, Huang Q, Zhang S, Zhang Q, Chang J, Qiu X and Wang E: Hsa-miR-125a-3p and hsa-miR-125a-5p are downregulated in non-small cell lung cancer and have inverse effects on invasion and migration of lung cancer cells. BMC Cancer 10 $318,2010$.

9. Ferretti E, De Smaele E, Po A, Di Marcotullio L, Tosi E, Espinola MS, Di Rocco C, Riccardi R, Giangaspero F, Farcomeni A, et al: MicroRNA profiling in human medulloblastoma. Int J Cancer 124: 568-577, 2009.

10. Hashiguchi Y, Nishida N, Mimori K, Sudo T, Tanaka F, Shibata K, Ishii H, Mochizuki H, Hase K, Doki Y, et al: Down-regulation of miR-125a-3p in human gastric cancer and its clinicopathological significance. Int J Oncol 40: 1477-1482, 2012

11. Nishida N, Mimori K, Fabbri M, Yokobori T, Sudo T, Tanaka F, Shibata K, Ishii H, Doki Y and Mori M: MicroRNA-125a-5p is an independent prognostic factor in gastric cancer and inhibits the proliferation of human gastric cancer cells in combination with trastuzumab. Clin Cancer Res 17: 2725-2733, 2011

12. Gerber HP, McMurtrey A, Kowalski J, Yan M, Keyt BA, Dixit V and Ferrara N: Vascular endothelial growth factor regulates endothelial cell survival through the phosphatidylinositol 3'-kinase/ Akt signal transduction pathway. Requirement for Flk-1/KDR activation. J Biol Chem 273: 30336-30343, 1998

13. Coultas L, Chawengsaksophak K and Rossant J: Endothelial cells and VEGF in vascular development. Nature 438: 937-945, 2005.

14. Caron C, Spring K, Laramée M, Chabot C, Cloutier M, Gu H and Royal I: Non-redundant roles of the Gab1 and Gab2 scaffolding adapters in VEGF-mediated signalling, migration, and survival of endothelial cells. Cell Signal 21: 943-953, 2009.

15. Elayappan B, Ravinarayannan H, Pasha SPBS, Lee KJ and Gurunathan S: PEDF inhibits VEGF- and EPO- induced angiogenesis in retinal endothelial cells through interruption of PI3K/ Akt phosphorylation. Angiogenesis 12: 313-324, 2009.

16. Radisavljevic Z, Avraham H and Avraham S: Vascular endothelial growth factor up-regulates ICAM-1 expression via the phosphatidylinositol $3 \mathrm{OH}$-kinase/AKT/Nitric oxide pathway and modulates migration of brain microvascular endothelial cells. J Biol Chem 275: 20770-20774, 2000.

17. Gerhardt H, Golding M, Fruttiger M, Ruhrberg C, Lundkvist A, Abramsson A, Jeltsch M, Mitchell C, Alitalo K, Shima D, et al: VEGF guides angiogenic sprouting utilizing endothelial tip cell filopodia. J Cell Biol 161: 1163-1177, 2003.

18. Adams RH and Alitalo K: Molecular regulation of angiogenesis and lymphangiogenesis. Nat Rev Mol Cell Biol 8: 464-478, 2007.

19. Herbert SP and Stainier DYR: Molecular control of endothelial cell behaviour during blood vessel morphogenesis. Nat Rev Mol Cell Biol 12: 551-564, 2011.

20. Wang X, Chen X, Fang J and Yang C: Overexpression of both VEGF-A and VEGF-C in gastric cancer correlates with prognosis, and silencing of both is effective to inhibit cancer growth. Int J Clin Exp Pathol 6: 586-597, 2013.

21. Zhao R, Liu XQ, Wu XP, Liu YF, Zhang ZY, Yang GY, Guo S, Niu J, Wang JY and Xu KS: Vascular endothelial growth factor (VEGF) enhances gastric carcinoma invasiveness via integrin alpha(v)beta6. Cancer Lett 287: 150-156, 2010.

22. Peng Z, Wei D, Wang L, Tang H, Zhang J, Le X, Jia Z, Li Q and Xie K: RUNX3 inhibits the expression of vascular endothelial growth factor and reduces the angiogenesis, growth, and metastasis of human gastric cancer. Clin Cancer Res 12: 6386-6394, 2006.

23. Ji YN, Wang Q, Li Y and Wang Z: Prognostic value of vascular endothelial growth factor A expression in gastric cancer: A metaanalysis. Tumour Biol 35: 2787-2793, 2014.
24. De Luca A, Lamura L, Gallo M, Maffia V and Normanno N: Mesenchymal stem cell-derived interleukin- 6 and vascular endothelial growth factor promote breast cancer cell migration. J Cell Biochem 113: 3363-3370, 2012

25. Yue X, Wang P, Xu J,Zhu Y, Sun G, Pang Q and Tao R: MicroRNA205 functions as a tumor suppressor in human glioblastoma cells by targeting VEGF-A. Oncol Rep 27: 1200-1206, 2012.

26. Wen J, Zhao Y, Li J, Weng C, Cai J, Yang K, Yuan H, ImperatoMcGinley J and Zhu YS: Suppression of DHT-induced paracrine stimulation of endothelial cell growth by estrogens via prostate cancer cells. Prostate 73: 1069-1081, 2013.

27. Vacca A, Ria R, Ribatti D, Semeraro F, Djonov V, Di Raimondo F and Dammacco F: A paracrine loop in the vascular endothelial growth factor pathway triggers tumor angiogenesis and growth in multiple myeloma. Haematologica 88: 176-185, 2003

28. Kinnaird T, Stabile E, Burnett MS, Lee CW, Barr S, Fuchs S and Epstein SE: Marrow-derived stromal cells express genes encoding a broad spectrum of arteriogenic cytokines and promote in vitro and in vivo arteriogenesis through paracrine mechanisms. Circ Res 94: 678-685, 2004.

29. Neviani P, Santhanam R, Oaks JJ, Eiring AM, Notari M, Blaser BW, Liu S, Trotta R, Muthusamy N, GambacortiPasserini C, et al: FTY720, a new alternative for treating blast crisis chronic myelogenous leukemia and Philadelphia chromosome-positive acute lymphocytic leukemia. J Clin Invest 117: 2408-2421, 2007.

30. Qu J, Lu W, Li B, Lu C and Wan X: WWOX induces apoptosis and inhibits proliferation in cervical cancer and cell lines. Int J Mol Med 31: 1139-1147, 2013.

31. Shao R, Xia W and Hung MC: Inhibition of angiogenesis and induction of apoptosis are involved in E1A-mediated bystander effect and tumor suppression. Cancer Res 60: 3123-3126, 2000.

32. Su SJ, Yeh TM, Chuang WJ, Ho CL, Chang KL, Cheng HL, Liu HS, Cheng HL, Hsu PY and Chow NH: The novel targets for anti-angiogenesis of genistein on human cancer cells. Biochem Pharmacol 69: 307-318, 2005.

33. Dallaglio K, Bruno A, Cantelmo AR, Esposito AI, Ruggiero L, Orecchioni S, Calleri A, Bertolini F, Pfeffer U, Noonan DM, et al: Paradoxic effects of metformin on endothelial cells and angiogenesis. Carcinogenesis 35: 1055-1066, 2014.

34. Zaytseva YY, Elliott VA, Rychahou P, Mustain WC, Kim JT, Valentino J, Gao T, O'Connor KL, Neltner JM, Lee EY, et al: Cancer cell-associated fatty acid synthase activates endothelial cells and promotes angiogenesis in colorectal cancer. Carcinogenesis 35: 1341-1351, 2014

35. Lu C and Tanigawa N: Spontaneous apoptosis is inversely related to intratumoral microvessel density in gastric carcinoma. Cancer Res 57: 221-224, 1997.

36. Hillen HF, Hak LE, Joosten-Achjanie SR and Arends JW: Microvessel density in unknown primary tumors. Int J Cancer 74: 81-85, 1997

37. Li XJ, Ren ZJ and Tang JH: MicroRNA-34a: A potential therapeutic target in human cancer. Cell Death Dis 5: e1327, 2014.

38. Blandino G, Fazi F, Donzelli S, Kedmi M, Sas-Chen A, Muti P, Strano S and Yarden Y: Tumor suppressor microRNAs: A novel non-coding alliance against cancer. FEBS Lett 588: 2639-2652, 2014.

39. Maroof H, Salajegheh A, Smith RA and Lam AKY: Role of microRNA-34 family in cancer with particular reference to cancer angiogenesis. Exp Mol Pathol 97: 298-304, 2014.

40. Yan JW, Lin JS and He XX: The emerging role of miR-375 in cancer. Int J Cancer 135: 1011-1018, 2014.

41. Koukourakis MI, Giatromanolaki A, Sivridis E, Gatter KC and Harris AL: High DLL4 expression in tumour-associated vessels predicts for favorable radiotherapy outcome in locally advanced squamous cell head-neck cancer (HNSCC). Angiogenesis 16: 343-351, 2013.

42. Weidner N, Folkman J, Pozza F, Bevilacqua P, Allred EN, Moore DH, Meli S and Gasparini G: Tumor angiogenesis: A new significant and independent prognostic indicator in early-stage breast carcinoma. J Natl Cancer Inst 84: 1875-1887, 1992.

43. Epstein RJ: VEGF signaling inhibitors: More pro-apoptotic than anti-angiogenic. Cancer Metastasis Rev 26: 443-452, 2007.

44. Liu F, You X, Wang Y, Liu Q, Liu Y, Zhang S, Chen L, Zhang X and Ye L: The oncoprotein HBXIP enhances angiogenesis and growth of breast cancer through modulating FGF8 and VEGF. Carcinogenesis 35: 1144-1153, 2014.

45. Shih YP, Liao YC, Lin Y and Lo SH: DLC1 negatively regulates angiogenesis in a paracrine fashion. Cancer Res 70: 8270-8275, 2010 . 
46. Kryza T, Achard C, Parent C, Marchand-Adam S, GuillonMunos A, Iochmann S, Korkmaz B, Respaud R, Courty Y and Heuzé-Vourc'h N: Angiogenesis stimulated by human kallikreinrelated peptidase 12 acting via a platelet-derived growth factor B-dependent paracrine pathway. FASEB J 28: 740-751, 2014.

47. Otrock ZK, Makarem JA and Shamseddine AI: Vascular endothelial growth factor family of ligands and receptors (review). Blood Cells Mol Dis 38: 258-268, 2007.

48. Hicklin DJ and Ellis LM: Role of the vascular endothelial growth factor pathway in tumor growth and angiogenesis. J Clin Oncol 23: 1011-1027, 2005.

49. Dayanir V, Meyer RD, Lashkari K and Rahimi N: Identification of tyrosine residues in vascular endothelial growth factor receptor-2/ FLK-1 involved in activation of phosphatidylinositol 3-kinase and cell proliferation. J Biol Chem 276: 17686-17692, 2001.
50. Pan Y, Wu Q, Qin L, Cai J and Du B: Gold nanoparticles inhibit VEGF165-induced migration and tube formation of endothelial cells via the Akt pathway. BioMed Res Int 2014: 418624, 2014.

51. Xu Y, Huang Z and Liu Y: Reduced miR-125a-5p expression is associated with gastric carcinogenesis through the targeting of E2F3. Mol Med Rep 10: 2601-2608, 2014.

52. Bi Q, Tang S, Xia L, Du R, Fan R, Gao L, Jin J, Liang S, Chen Z, $\mathrm{Xu}$ G, et al: Ectopic expression of MiR-125a inhibits the proliferation and metastasis of hepatocellular carcinoma by targeting MMP11 and VEGF. PLoS One 7: e40169, 2012.

53. Cao Y: VEGF-targeted cancer therapeutics-paradoxical effects in endocrine organs. Nat Rev Endocrinol 10: 530-539, 2014.

54. Jain RK: Antiangiogenesis strategies revisited: From starving tumors to alleviating hypoxia. Cancer Cell 26: 605-622, 2014. 